\title{
The onset of convection in rotating circular cylinders with experimental boundary conditions
}

\author{
KEKE ZHANG ${ }^{1} \uparrow$ AND XINHAO $\mathrm{LIAO}^{2}$ \\ ${ }^{1}$ Department of Mathematical Sciences, University of Exeter, Exeter EX4 4QE, UK \\ ${ }^{2}$ Shanghai Astronomical Observatory, Chinese Academy of Sciences, \\ Shanghai 200030, China
}

(Received 1 August 2008 and in revised form 27 October 2008)

Convective instabilities in a fluid-filled circular cylinder heated from below and rotating about its vertical axis are investigated both analytically and numerically under experimental boundary conditions. It is found that there exist two different forms of convective instabilities: convection-driven inertial waves for small and moderate Prandtl numbers and wall-localized travelling waves for large Prandtl numbers. Asymptotic solutions for both forms of convection are derived and numerical simulations for the same problem are also performed, showing a satisfactory quantitative agreement between the asymptotic and numerical analyses.

\section{Introduction}

Motivated by the wish to understand the fundamental dynamics taking place in planetary fluid interiors and atmospheres, convection in rotating cylindrical geometry has been extensively studied both experimentally and theoretically (see, for example, Daniels 1980; Zhong, Ecke \& Steinberg 1991; Herrmann \& Busse 1993; Kuo \& Cross 1993; Aurnou et al. 2003; Plaut 2003; Young \& Read 2008). In particular, careful numerical studies of convective instabilities in a fluid contained in a circular cylinder uniformly heated from below and rotating about its vertical axis (Goldstein et al. 1993, 1994) reveal an exceedingly complicated and seemingly incomprehensible behaviour at small and moderate Prandtl numbers.

We have recently undertaken the study of convective instabilities in a rotating circular cylinder with stress-free ends (Zhang, Liao \& Busse 2007). The assumption of the stress-free boundary condition permits separable solutions and, consequently, leads to a much simplified mathematical problem governed by ordinary differential equations. It reveals that inertial convection is preferred for fluids with small and moderate Prandtl numbers if the stress-free condition is assumed. Since inertial convection is energetically driven by thermal buoyancy against viscous dissipation that occurs largely in the viscous boundary layers (see, for example, Zhang 1995; Busse \& Simitev 2004), the type of velocity boundary condition plays, both physically and mathematically, an essential role in determining the main properties of convection. The Ekman boundary layers resulting from the non-slip top and bottom not only introduce strong mass fluxes into the interior but also bring dominant terms in the asymptotic 
solution of inertial convection in rapidly rotating cylinders. It is therefore desirable to gain a better understanding of convection under experimental boundary conditions, the problem then being governed by partial differential equations, providing a useful theoretical framework and guidance for experimental studies of the problem.

Thus the primary objective of this study is to understand, through both numerical and asymptotic analysis, convective instabilities in a rotating cylinder under experimental boundary conditions. Our focus will be mainly on instabilities at small and moderate Prandtl numbers. However, the asymptotic solution for large Prandtl numbers is also briefly discussed for the purpose of completeness. It is fortunate that on recognizing and utilizing the physical nature of convective instabilities, we are able to derive several relatively simple asymptotic formulas that correctly describe the key features of convection for all values of the Prandtl number and the aspect ratio and which agree satisfactorily with the results of numerical analysis.

In what follows we shall begin by presenting the mathematical equations in $\S 2$. The numerical analysis is briefly presented in $\S 3$, while the corresponding asymptotic analysis is discussed in $\S 4$, with a summary and several remarks given in $\S 5$.

\section{Mathematical formulation}

Consideration is given to convective instabilities in a Boussinesq fluid confined in a circular cylinder of radius $s_{o}$ and height $d$ with constant thermal diffusivity $\kappa$, thermal expansion coefficient $\alpha$ and kinematic viscosity $\nu$. The circular cylinder rotates uniformly about its axis with a constant vertical angular velocity $\Omega$ in the presence of a constant gravitational field $g=-g \hat{z}$, and is heated uniformly from below to produce an unstable vertical temperature gradient, $\nabla T_{0}=-\beta \hat{z}$, where $\beta$ is a positive constant. Cylindrical coordinates $(s, \phi, z)$ with the corresponding unit vectors $(\hat{\boldsymbol{s}}, \hat{\boldsymbol{\phi}}, \hat{z}$, where $\hat{z}$ is parallel to the axis of rotation) are used. Making use of the depth $d$ as the length scale, $\Omega^{-1}$ as the unit of time and $\beta d^{3} \Omega / \kappa$ as the unit of temperature fluctuations, the problem of linear convective instability is governed by the equations

$$
\begin{gathered}
\frac{\partial \boldsymbol{u}}{\partial t}+2 \hat{z} \times \boldsymbol{u}=-\nabla p+R \Theta \hat{z}+E \nabla^{2} \boldsymbol{u}, \\
\nabla \cdot \boldsymbol{u}=0, \\
\operatorname{Pr} \frac{\partial \Theta}{\partial t}=E\left(\hat{z} \cdot \boldsymbol{u}+\nabla^{2} \Theta\right),
\end{gathered}
$$

where $\boldsymbol{u}$ is the three-dimensional velocity field, and the temperature deviation from the purely conductive state $T_{0}(z)$ is represented by $\Theta$. In cylindrical coordinates, we write solutions of the equations in the form $(\boldsymbol{u}, \Theta)(s, \phi, z, t)=(\boldsymbol{u}, \Theta)$ $(s, z) \exp [\mathrm{i}(2 \sigma t+m \phi)]$, where $m$ is the azimuthal wavenumber assumed to be positive and $\sigma$ is the half-frequency which is used because of its property $|\sigma|<1$ in the limit $E=0$. The three non-dimensional physical parameters - the Rayleigh number $R$, the Prandtl number $\operatorname{Pr}$ and the Ekman number $E$ - are defined as

$$
R=\frac{\alpha \beta g d^{2}}{\Omega \kappa}, \quad \operatorname{Pr}=\frac{\nu}{\kappa}, \quad E=\frac{\nu}{\Omega d^{2}} .
$$

The geometric parameter is given by the aspect ratio $\Gamma$, defined as $\Gamma=s_{0} / d$. Appropriately for experiments of the problem (see, for example, Goldstein et al. 1993), we shall assume no-slip velocity and perfectly conducting conditions on the 
top and bottom of the cylinder,

$$
\boldsymbol{u}=\Theta=0 \quad \text { at } \quad z=0,1 .
$$

On the sidewall, we assume no-slip and perfectly insulating conditions which require

$$
\boldsymbol{u}=\frac{\partial \Theta}{\partial s}=0 \quad \text { at } \quad s=\Gamma .
$$

Equations (2.1)-(2.3) subject to the boundary conditions in (2.4)-(2.5) form the convective instability problem which will be solved by both asymptotic and numerical analysis at, as in practical computations and physical experiments, a small but fixed $E$ for different values of $\operatorname{Pr}$.

\section{Numerical analysis}

Compared to the numerical analysis for stress-free ends, which allows solutions in separable variables, the numerical analysis for the no-slip condition requires solutions of partial differential equations and hence is much more complicated. In order to determine the most unstable mode of convective instabilities, we must consider disturbances of all possible azimuthal wavenumbers. Axisymmetric convection may be physically preferred in rotating spherical systems (see, for example, Net, Carcia \& Sánchez 2008). However, our extensive numerical and asymptotic studies show that axisymmetric $(m=0)$ instabilities are not preferred for any values of $P r$ when $E \ll 1$ and $\Gamma=O(1)$. In consequence, we shall present the numerical and asymptotic analysis only for non-axisymmetric $(m \neq 0)$ solutions.

For non-axisymmetric instabilities with $m \neq 0$, the velocity vector $\boldsymbol{u}$ satisfying (2.2) can be expressed in terms of two scalar potentials $\Psi$ and $\Phi$ (Marqués 1990):

$$
\boldsymbol{u}=\frac{1}{s} \frac{\partial \Psi}{\partial \phi} \hat{\boldsymbol{s}}+\left(\frac{\partial \Phi}{\partial z}-\frac{\partial \Psi}{\partial s}\right) \hat{\boldsymbol{\phi}}-\frac{1}{s} \frac{\partial \Phi}{\partial \phi} \hat{z}
$$

In terms of $\Psi$ and $\Phi$, the non-slip condition on the sidewall and the ends becomes

$$
\Psi=\frac{\partial \Psi}{\partial s}=\Phi=0 \quad \text { at } \quad s=\Gamma ; \Psi=\frac{\partial \Phi}{\partial z}=\Phi=0 \quad \text { at } \quad z=0,1
$$

Making use of the expression (3.1) and applying $\hat{z} \cdot \nabla \times$ and $\hat{s} \cdot \nabla \times$ onto (2.1), we can derive the three independent non-dimensional scalar equations:

$$
\begin{gathered}
\left(\frac{\partial}{\partial t}-E \nabla^{2}\right)\left[\frac{1}{s} \frac{\partial}{\partial s}\left(s \frac{\partial \Phi}{\partial z}\right)-\left(\nabla^{2}-\frac{\partial^{2}}{\partial z^{2}}\right) \Psi\right]+\frac{2}{s} \frac{\partial^{2} \Phi}{\partial z \partial \phi}=0, \\
{\left[\frac{\partial}{\partial t}-E\left(\nabla^{2}+\frac{2}{s} \frac{\partial}{\partial s}+\frac{1}{s^{2}}\right)\right]\left[\frac{\partial^{2} \Psi}{\partial s \partial z}-\left(\nabla^{2}-\frac{1}{s} \frac{\partial}{\partial s} s \frac{\partial}{\partial s}\right) \Phi\right]} \\
-\frac{2}{s} \frac{\partial^{2} \Psi}{\partial z \partial \phi}-\frac{2 E}{s}\left[\frac{1}{s} \frac{\partial}{\partial s}\left(s \frac{\partial^{2} \Phi}{\partial z^{2}}\right)-\left(\nabla^{2}-\frac{\partial^{2}}{\partial z^{2}}\right) \frac{\partial \Psi}{\partial z}\right]-\frac{R}{s} \frac{\partial \Theta}{\partial \phi}=0, \\
{\left[E \nabla^{2}-\operatorname{Pr} \frac{\partial}{\partial t}\right] \Theta-\frac{E}{s} \frac{\partial \Phi}{\partial \phi}=0 .}
\end{gathered}
$$

The above equations are then solved numerically by using the Galerkin-type method in which, for example, $\Psi$ and $\Phi$ are expanded in terms of the Chebyshev functions 


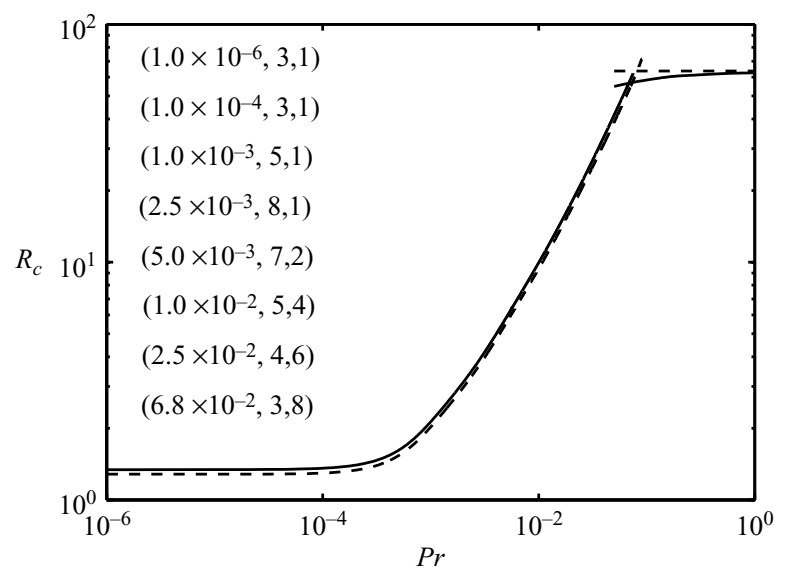

FIGURE 1. The critical Rayleigh number $R_{c}$ is plotted as a function of $P r$ in a rotating cylinder with $\Gamma=2$ and $E=10^{-4}$. Solid lines represent the numerical solutions while dashed lines result from the asymptotic solutions. Of the three numbers in the bracket, the first is $\mathrm{Pr}$, the second is $m$ and the third is the number of nodes in the $s$ direction.

$T_{j}(x)$ :

$$
\begin{aligned}
& \Psi=s^{m}(1-\check{s})^{2}\left(1-\check{z}^{2}\right)\left[\sum_{j=0}^{J} \sum_{l=0}^{L} \Psi_{m j l} T_{j}(\check{z}) T_{l}(\check{s})\right] \mathrm{e}^{\mathrm{i}(m \phi+2 \sigma t)}, \\
& \Phi=s^{m+1}(1-\check{s})\left(1-\check{z}^{2}\right)^{2}\left[\sum_{j=0}^{J} \sum_{l=0}^{L} \Phi_{m j l} T_{j}(\check{z}) T_{l}(\check{s})\right] \mathrm{e}^{\mathrm{i}(m \phi+2 \sigma t)},
\end{aligned}
$$

where $\check{z}=2 z-1, \check{s}=2 s / \Gamma-1$ and $J=L=O(100)$ for achieving an accuracy within $1 \%$, the factors $s^{m}$ and $s^{m+1}$ are imposed for regularity of the solution at $s=0$ and the complex coefficients like $\Psi_{m j l}$ are obtained, together with the Rayleigh number $R$ and the half-frequency $\sigma$, by a standard numerical procedure.

The primary aim of our numerical analysis is to provide a valuable comparison with the results of the asymptotic analysis valid only for small $E$. Some typical results of the numerical analysis, along with the asymptotic results, are presented in figure 1 , showing the critical Rayleigh number $R_{c}$ for the most unstable mode of convective instabilities as a function of $P r$ in a rotating cylinder with $\Gamma=2$ and $E=10^{-4}$. The convection patterns for three typical Prandtl numbers, $P r=0.0025,0.025$ and 1.0, are depicted in figure 2. We shall discuss the relevant details when comparing them with the asymptotic solutions.

\section{Asymptotic analysis}

A key assumption in the asymptotic analysis is that the velocity of convection at leading order for small or moderate $\operatorname{Pr}$ can be represented by a single inertial-wave mode while buoyancy forces appear only at the next order to drive the inertial wave against the effects of viscous damping, leading to an asymptotic expansion in the form

$$
\left.\begin{array}{ll}
\boldsymbol{u}=\boldsymbol{u}_{0}+\boldsymbol{u}_{b}+\boldsymbol{u}_{1}, & p=p_{0}+p_{b}+p_{1}, \\
\sigma=\sigma_{0}+\sigma_{1}, & \Theta=\Theta_{0}+\cdots, \quad R=R_{1}+\cdots,
\end{array}\right\}
$$

where $\boldsymbol{u}_{1}, p_{1}$ and $\sigma_{1}$ represent small interior viscous corrections to the leading-order inviscid interior solution $\boldsymbol{u}_{0}, p_{0}$ and $\sigma_{0}$, while $\boldsymbol{u}_{b}$ and $p_{b}$ denote solutions of the viscous boundary layers which are non-zero only in the vicinity of the bounding surface of 

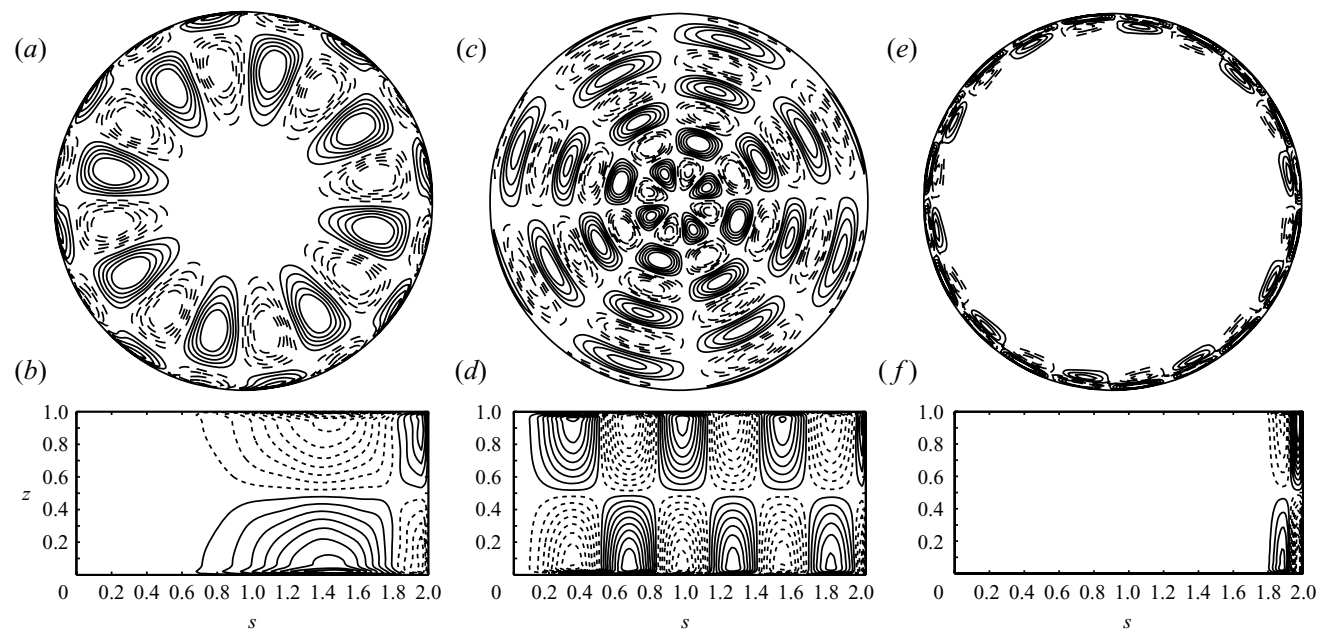

Figure 2. Contours of $u_{\phi}$ in a horizontal plane at $z=0.75$ (upper panels) and in a vertical plane (lower panels): $(a, b)$ for $\operatorname{Pr}=0.0025,(c, d)$ for $\operatorname{Pr}=0.025$ and $(e, f)$ for $\operatorname{Pr}=1.0$ with $\Gamma=2$ and $E=10^{-4}$.

the cylinder. More precisely, $\left|\boldsymbol{u}_{0}\right|=O(1),\left|\boldsymbol{u}_{b}\right|=O(1)$ and $\left|\boldsymbol{u}_{1}\right| \ll 1$, with the flux from the oscillatory boundary layer $\boldsymbol{u}_{b}$ providing the matching condition for the secondary interior flow $\boldsymbol{u}_{1}$. It is of importance to note that $E^{1 / 2}$ cannot be used as an expansion parameter in this problem because the thickness of an oscillatory viscous boundary layer is not generally of order $E^{1 / 2}$, the flux from the oscillatory boundary layer is not generally of order $E^{1 / 2}$ and the internal viscous contribution is not generally of the order $E^{1 / 2}$ smaller than that of the oscillatory boundary layer (Zhang \& Liao 2008). In other words, the effects of spatial and temporal non-uniformities obscure the form of the asymptotic expansion even for the first viscous corrective terms.

After substitution of the expansion into (2.1)-(2.3), the leading-order problem, subject to the usual inviscid boundary condition, is given by

$$
\begin{gathered}
\frac{\partial \boldsymbol{u}_{0}}{\partial t}+2 \hat{z} \times \boldsymbol{u}_{0}+\nabla p_{0}=0, \\
\nabla \cdot \boldsymbol{u}_{0}=0, \\
\operatorname{Pr} \frac{\partial \Theta_{0}}{\partial t}=E\left(\hat{z} \cdot \boldsymbol{u}_{0}+\nabla^{2} \Theta_{0}\right),
\end{gathered}
$$

which describe non-dissipative thermal inertial waves with solutions in the form

$$
\begin{aligned}
\hat{\boldsymbol{s}} \cdot \boldsymbol{u}_{0} & =\frac{-\mathrm{i}}{2\left(1-\sigma_{0}^{2}\right)}\left[\frac{\sigma_{0} \xi}{\Gamma} J_{m-1}(\tilde{s})+\frac{m\left(1-\sigma_{0}\right)}{s} J_{m}(\tilde{s})\right] \cos (\pi z) \mathrm{e}^{\mathrm{i}\left(m \phi+2 \sigma_{0} t\right)}, \\
\hat{\boldsymbol{\phi}} \cdot \boldsymbol{u}_{0} & =\frac{1}{2\left(1-\sigma_{0}^{2}\right)}\left[\frac{\xi}{\Gamma} J_{m-1}(\tilde{s})-\frac{m\left(1-\sigma_{0}\right)}{s} J_{m}(\tilde{s})\right] \cos (\pi z) \mathrm{e}^{\mathrm{i}\left(m \phi+2 \sigma_{0} t\right)}, \\
\hat{z} \cdot \boldsymbol{u}_{0} & =\frac{-\mathrm{i} \pi}{2 \sigma_{0}} J_{m}(\tilde{s}) \sin (\pi z) \mathrm{e}^{\mathrm{i}\left(m \phi+2 \sigma_{0} t\right)}, \\
\Theta_{0} & =\sum_{n=1}^{N} \frac{-2 \mathrm{i} m\left(\xi^{2}-\beta_{m n}^{2}\right) \mathscr{Q}_{m n} J_{m}(\xi) \sin (\pi z)}{\pi \Gamma^{2}\left[\pi^{2}+\left(\beta_{m n} / \Gamma\right)^{2}+2 P r E^{-1} \sigma_{0} i\right]} \frac{J_{m}\left(\beta_{m n} s / \Gamma\right)}{J_{m}\left(\beta_{m n}\right)} \mathrm{e}^{\mathrm{i}\left(m \phi+2 \sigma_{0} t\right)}, \\
p_{0} & =J_{m}(\tilde{s}) \cos (\pi z) \mathrm{e}^{\mathrm{i}\left(m \phi+2 \sigma_{0} t\right)},
\end{aligned}
$$


where $\tilde{s}=\xi s / \Gamma$ (the simplest vertical structure that is relevant to convective instabilities is taken), $J_{m}(x)$ denotes the standard Bessel function, $\beta_{m n}$ are the roots of $J_{m}^{\prime}\left(\beta_{m n}\right)=0$ with $0<\beta_{m 1}<\beta_{m 2}<\beta_{m 3} \cdots, \mathscr{Q}_{m n}=\left(\pi \Gamma \beta_{m n}\right)^{2} /\left[2 \sigma_{0}^{2}\left(\xi^{2}-\beta_{m n}^{2}\right)^{2}\left(\beta_{m n}^{2}-m^{2}\right)\right]$, $N \leqslant O(10)$ for achieving an accuracy within $1 \%$ and $\xi$ is a solution of

$$
\begin{gathered}
\xi J_{m-1}(\xi)+\frac{\sigma_{0}}{\left|\sigma_{0}\right|}\left\{\left[1+\left(\frac{\xi}{\Gamma \pi}\right)^{2}\right]^{1 / 2}-1\right\} m J_{m}(\xi)=0, \\
\sigma_{0}= \pm\left[1+(\xi / \Gamma \pi)^{2}\right]^{-1 / 2} .
\end{gathered}
$$

Different values of $\xi$ or $\sigma_{0}$ correspond to different inertial modes.

At the next order, the equations governing the perturbations of the interior flow are

$$
\begin{aligned}
\mathrm{i} 2 \sigma_{0} \boldsymbol{u}_{1}+2 \hat{z} \times \boldsymbol{u}_{1}+\nabla p_{1} & =R_{1} \hat{z} \Theta_{0}+E \nabla^{2} \boldsymbol{u}_{0}-\mathrm{i} 2 \sigma_{1} \boldsymbol{u}_{0}, \\
\nabla \cdot \boldsymbol{u}_{1} & =0 .
\end{aligned}
$$

It is straightforward to derive the solvability condition required for the inhomogeneous equation (4.12):

$$
\begin{array}{r}
E^{1 / 2} \int_{S} p_{0}^{*}\left[\int_{0}^{\infty} \hat{\boldsymbol{n}} \cdot \nabla \times\left(\hat{\boldsymbol{n}} \times \boldsymbol{u}_{b}\right) \mathrm{d} \eta\right] \mathrm{d} S=\int_{V}\left[R_{1} \boldsymbol{u}_{0}^{*} \cdot \hat{z} \Theta_{0}-2 \mathrm{i} \sigma_{1}\left|\boldsymbol{u}_{0}\right|^{2}\right. \\
\left.-E\left|\nabla \times \boldsymbol{u}_{0}\right|^{2}\right] \mathrm{d} V,
\end{array}
$$

where $\boldsymbol{u}_{0}^{*}$ and $p_{0}^{*}$ denote the complex conjugates of $\boldsymbol{u}_{0}$ and $p_{0}, \int_{V}$ represents a volume integral over the cylinder, $\int_{S}$ represents a surface integral over its bounding surface with the normal vector $\hat{\boldsymbol{n}}$ and $\eta$ is the stretched boundary-layer coordinate. In deriving (4.14), the mass flux from the Ekman boundary layer is used to provide the matching condition for $\boldsymbol{u}_{1}$ at the outer edge of the layer. Each integral in (4.14) can be evaluated. Upon using (4.5)-(4.8) together with the properties of the Bessel function, we can show that

$$
\begin{aligned}
\int_{V} \boldsymbol{u}_{0}^{*} \cdot \hat{z} \Theta_{0} \mathrm{~d} V & =\sum_{n=1}^{N} \frac{\pi \mathscr{Q}_{m n}}{\pi^{2}+\left(\beta_{m n} / \Gamma\right)^{2}+2 \mathrm{i} \operatorname{Pr} E^{-1} \sigma_{0}}\left[\frac{m J_{m}(\xi)}{\sigma_{0}}\right]^{2}, \\
\int_{V}\left|\boldsymbol{u}_{0}\right|^{2} \mathrm{~d} V & =\frac{\sigma_{0}^{2}}{\pi^{2}} \int_{V}\left|\nabla \times \boldsymbol{u}_{0}\right|^{2} \mathrm{~d} V=\frac{\pi\left[(\pi \Gamma)^{2}+m\left(m-\sigma_{0}\right)\right] J_{m}^{2}(\xi)}{4 \sigma_{0}^{2}\left(1-\sigma_{0}^{2}\right)} .
\end{aligned}
$$

In order to evaluate the surface integral on the left-hand side of (4.14), we must derive the boundary-layer flow $\boldsymbol{u}_{b}$ on the bounding surface of a cylinder. Consider first the Ekman boundary layer on the bottom surface at $z=0$ described by

$$
\left(\mathrm{i} 2 \sigma_{0}-\frac{\partial^{2}}{\partial \eta^{2}}\right) \hat{z} \times \boldsymbol{u}_{b}=2 \boldsymbol{u}_{b}, \quad\left(\mathrm{i} 2 \sigma_{0}-\frac{\partial^{2}}{\partial \eta^{2}}\right) \boldsymbol{u}_{b}=-2 \hat{z} \times \boldsymbol{u}_{b},
$$

where $\eta=E^{-1 / 2} z$ and $\boldsymbol{u}_{b}=-\boldsymbol{u}_{0}$ at $z=0$ so that the no-slip condition is obeyed. Note that $\eta=0$ is at the bottom surface while $\eta=\infty$ is at the outer edge of the boundary layer but still located at the bottom in terms of the coordinate $z$. The boundary-layer solution to (4.17) on the bottom surface can be expressed in the form

$$
\begin{aligned}
\boldsymbol{u}_{b}= & \frac{1}{4\left(1-\sigma_{0}^{2}\right)}\left\{\left[\frac{\xi\left(\sigma_{0}-1\right)}{\Gamma} J_{m-1}(\tilde{s})+\frac{2 m\left(1-\sigma_{0}\right)}{s} J_{m}(\tilde{s})\right](\mathrm{i} \hat{\boldsymbol{s}}+\hat{\boldsymbol{\phi}}) \mathrm{e}^{-(1+\mathrm{i}) \eta \sqrt{1+\sigma_{0}}}\right. \\
& \left.+\left[\frac{\xi\left(\sigma_{0}+1\right)}{\Gamma} J_{m-1}(\tilde{s})\right](\mathrm{i} \hat{\boldsymbol{s}}-\hat{\boldsymbol{\phi}}) \mathrm{e}^{-(1-\mathrm{i}) \eta \sqrt{1-\sigma_{0}}}\right\} \mathrm{e}^{\mathrm{i}\left(m \phi+2 \sigma_{0} t\right)}
\end{aligned}
$$


by which, together with (4.9), we can obtain the contribution from the viscous boundary layer at $z=0$ to the integral on the left-hand side of (4.14):

$$
\begin{aligned}
& \int_{0}^{2 \pi} \int_{0}^{\Gamma}\left(p_{0}^{*}\right)_{z=0}\left[\int_{0}^{\infty} \hat{\boldsymbol{n}} \cdot \nabla \times\left(\hat{\boldsymbol{n}} \times \boldsymbol{u}_{b}\right) \mathrm{d} \eta\right] s \mathrm{~d} s \mathrm{~d} \phi=\left[\frac{\pi J_{m}^{2}(\xi)}{8 \sigma_{0}^{2}\left(1-\sigma_{0}^{2}\right)}\right] \\
& \quad \times\left\{\left(\sqrt{1+\sigma_{0}}+\sqrt{1-\sigma_{0}}\right) \mathscr{C}_{1}-2 \sigma_{0}\left(\sqrt{1+\sigma_{0}}-\sqrt{1-\sigma_{0}}\right) \mathscr{C}_{2}\right. \\
& \left.+\mathrm{i}\left[\left(\sqrt{1+\sigma_{0}}-\sqrt{1-\sigma_{0}}\right) \mathscr{C}_{1}-2 \sigma_{0}\left(\sqrt{1+\sigma_{0}}+\sqrt{1-\sigma_{0}}\right) \mathscr{C}_{2}\right]\right\},
\end{aligned}
$$

where $\mathscr{C}_{1}=\left(1+\sigma_{0}^{2}\right)\left(m^{2}+\pi^{2} \Gamma^{2}\right)-2 m \sigma_{0}$ and $\mathscr{C}_{2}=\pi^{2} \Gamma^{2}+m\left(m-\sigma_{0}\right)$. Because of the symmetry between the top and the bottom of the cylinder, the contribution from the viscous boundary layer at $z=1$ is exactly the same as that from the boundary layer at $z=0$. Similarly, we can derive the contribution from the sidewall boundary layer to the integral on the left-hand side of (4.14):

$$
\begin{gathered}
\int_{0}^{2 \pi} \int_{0}^{1}\left(p_{0}^{*}\right)_{s=\Gamma}\left[\int_{0}^{\infty} \hat{\boldsymbol{n}} \cdot \nabla \times\left(\hat{\boldsymbol{n}} \times \boldsymbol{u}_{b}\right) \mathrm{d} \eta\right] \Gamma \mathrm{d} z \mathrm{~d} \phi \\
=\frac{\pi}{4 \Gamma \sigma_{0} \sqrt{\left|\sigma_{0}\right|}}\left(\mathrm{i}+\frac{\sigma_{0}}{\left|\sigma_{0}\right|}\right)\left(m^{2}+\Gamma^{2} \pi^{2}\right) J_{m}^{2}(\xi) .
\end{gathered}
$$

Substitution of (4.15), (4.16), (4.19) and (4.20) into the solvability condition (4.14) yields a complex equation whose real part determines $R_{1}$ while the imaginary part gives rise to the viscous correction $\sigma_{1}$ :

$$
\begin{aligned}
R_{1}= & \frac{E^{1 / 2}}{4 m^{2} \sqrt{1-\sigma_{0}^{2}}}\left[\sum_{n=1}^{N} \frac{\left(\pi^{2}+\beta_{m n}^{2} / \Gamma^{2}\right) \mathscr{Q}_{m n}}{\left(\pi^{2}+\beta_{m n}^{2} / \Gamma^{2}\right)^{2}+\left(2 \sigma_{0} \operatorname{Pr} / E\right)^{2}}\right]^{-1} \\
& \times\left\{\frac{E^{1 / 2} \pi^{2}\left[(\pi \Gamma)^{2}+m\left(m-\sigma_{0}\right)\right]}{\sigma_{0}^{2} \sqrt{1-\sigma_{0}^{2}}}-2 \sigma_{0} m\left[\sqrt{1+\sigma_{0}}+\sqrt{1-\sigma_{0}}\right]\right. \\
& \left.+\left(m^{2}+\pi^{2} \Gamma^{2}\right)\left[\frac{\sqrt{\left|\sigma_{0}\right|\left(1-\sigma_{0}^{2}\right)}}{\Gamma}+\left(1+\sigma_{0}\right)^{3 / 2}+\left(1-\sigma_{0}\right)^{3 / 2}\right]\right\} \\
2 \sigma= & 2 \sigma_{0}-\frac{\left(1-\sigma_{0}^{2}\right)^{1 / 2} E^{1 / 2}}{\pi^{2} \Gamma^{2}+m\left(m-\sigma_{0}\right)}\left\{\left[\sum_{n=1}^{N} \frac{\mathscr{Q}_{m n}}{\left(\pi^{2}+\beta_{m n}^{2} / \Gamma^{2}\right)^{2}+\left(2 \sigma_{0} \operatorname{Pr} / E\right)^{2}}\right]\right. \\
& \times\left[8 m^{2} R_{0} \operatorname{Pr}^{-3 / 2} \sigma_{0}\left(1-\sigma_{0}^{2}\right)^{1 / 2}\right]+2 \sigma_{0} m\left[\sqrt{1+\sigma_{0}}-\sqrt{1-\sigma_{0}}\right] \\
& \left.+\left(m^{2}+\pi^{2} \Gamma^{2}\right)\left[\frac{\sigma_{0} \sqrt{1-\sigma_{0}^{2}}}{\Gamma \sqrt{\left|\sigma_{0}\right|}}-\left(1+\sigma_{0}\right)^{3 / 2}+\left(1-\sigma_{0}\right)^{3 / 2}\right]\right\}
\end{aligned}
$$

In addition to the expressions for the Rayleigh number $R_{1}$ and the frequency $\sigma$, the leading-order velocity of convection can be explicitly written as 


$$
\begin{aligned}
\hat{\boldsymbol{s}} \cdot \boldsymbol{u}= & \frac{\mathrm{i}}{4\left(1-\sigma_{0}^{2}\right)}\left\{-2\left[\frac{\sigma_{0} \xi}{\Gamma} J_{m-1}(\tilde{s})+\frac{m\left(1-\sigma_{0}\right)}{s} J_{m}(\tilde{s})\right] \cos \pi z\right. \\
& +\left[\frac{\xi\left(\sigma_{0}-1\right)}{\Gamma} J_{m-1}(\tilde{s})+\frac{2 m\left(1-\sigma_{0}\right)}{s} J_{m}(\tilde{s})\right]\left[\mathrm{e}^{-\chi^{+} z}-\mathrm{e}^{-\chi^{+}(1-z)}\right] \\
& \left.+\frac{\xi\left(\sigma_{0}+1\right)}{\Gamma} J_{m-1}(\tilde{s})\left[\mathrm{e}^{-\chi^{-} z}-\mathrm{e}^{-\chi^{-}(1-z)}\right]\right\} \mathrm{e}^{\mathrm{i}(2 \sigma t+m \phi)}, \\
\hat{\boldsymbol{\phi}} \cdot \boldsymbol{u}= & \frac{1}{4\left(1-\sigma_{0}^{2}\right)}\left\{2\left[\frac{\xi J_{m-1}(\tilde{s})}{\Gamma}-\frac{m\left(1-\sigma_{0}\right)}{s} J_{m}(\tilde{s})\right] \cos \pi z+\frac{m J_{m}(\tilde{s})}{2 \sigma_{0} \Gamma} \cos \pi z \mathrm{e}^{-\chi(\Gamma-s)}\right. \\
& +\left[\frac{\xi\left(\sigma_{0}-1\right)}{\Gamma} J_{m-1}(\tilde{s})+\frac{2 m\left(1-\sigma_{0}\right)}{s} J_{m}(\tilde{s})\right]\left[\mathrm{e}^{-\chi^{+} z}-\mathrm{e}^{-\chi^{+}(1-z)}\right] \\
& \left.-\frac{\xi\left(\sigma_{0}+1\right)}{\Gamma} J_{m-1}(\tilde{s})\left[\mathrm{e}^{-\chi^{-} z}-\mathrm{e}^{-\chi^{-}(1-z)}\right]\right\} \mathrm{e}^{\mathrm{i}(2 \sigma t+m \phi)}, \\
\hat{\boldsymbol{z}} \cdot \boldsymbol{u}= & -\frac{\mathrm{i} \pi}{2 \sigma_{0}}\left[J_{m}(\tilde{s})-J_{m}(\xi) \mathrm{e}^{-\chi(\Gamma-s)}\right] \sin (\pi z) \mathrm{e}^{\mathrm{i}(2 \sigma t+m \phi)},
\end{aligned}
$$

where $\chi^{+}=(1+\mathrm{i}) \sqrt{E^{-1}\left(1+\sigma_{0}\right)}, \chi^{-}=(1-\mathrm{i}) \sqrt{E^{-1}\left(1-\sigma_{0}\right)}$ and $\chi=\sqrt{\left|\sigma_{0}\right| E^{-1}}(1+$ $\left.\mathrm{i} \sigma_{0} /\left|\sigma_{0}\right|\right)$. A complete analytical solution of the problem is described by (4.21)-(4.25).

As indicated by (4.21), the Rayleigh number $R_{1}$ required to initiate inertial convection increases rapidly with increasing $P r$ and the sidewall-localized convection would become physically preferred at large $\mathrm{Pr}$. It is worth mentioning that the asymptotic analysis (Herrmann \& Busse 1993) was concerned with the leading-order solution for which the effect of the non-slip condition was not considered. Since cylindrical geometry plays an insignificant role in the wall-localized convective flow, we can simply re-scale the asymptotic solution in a channel obtained by Liao, Zhang \& Chang (2006), giving rise to

$$
\begin{aligned}
& R=2 \pi^{2} \sqrt{6 \sqrt{3}}+73.80 E^{1 / 3} \\
& \sigma=\left[\pi^{2} \sqrt{3} \sqrt{2+\sqrt{3}}\right] E / \operatorname{Pr}-290.6 E^{4 / 3} \operatorname{Pr}^{-1}, \\
& m=\Gamma\left[\pi \sqrt{2+\sqrt{3}}-27.76 E^{1 / 3}\right],
\end{aligned}
$$

which has taken into account the non-slip boundary condition while its leading order is the same as that given by Herrmann \& Busse (1993).

For any given values of $\Gamma, P r$ and $E$, the following three steps are usually required to find the most unstable mode of convective instabilities: $(a)$ use (4.21) to calculate the smallest $R_{1}$ required for the non-axisymmetric inertial convection over different values of $\sigma_{0}$, i.e. over different inertial modes; $(b)$ use (4.26) to calculate the second Rayleigh number required for the wall-localized convection and $(c)$ compare the two Rayleigh numbers obtained at exactly the same $\Gamma, P r$ and $E$. Of physical significance is the minimum value of the Rayleigh number, which is denoted by $R_{c}$ and will be referred to as the critical Rayleigh number, at which convective instabilities first set in when the Rayleigh number $R$ gradually increases from zero.

The $R_{c}$ as a function of $P r$, calculated from both the asymptotic and the numerical analysis, is shown in figure 1 for $E=10^{-4}$ and $\Gamma=2$ : all the most unstable modes of convection are non-axisymmetric. The azimuthal wavenumber 
$m_{c}$ and the corresponding nodes in the radial direction for several typical Prandt numbers are also shown in figure 1. For example, the asymptotic formulas (4.21)(4.22) at $\operatorname{Pr}=0.0025$ yield $R_{c}=3.41, m_{c}=8, \sigma_{c}=0.474$ with $\sigma_{0}=0.4773$, while the numerical analysis gives $R_{c}=3.58, m_{c}=8, \sigma_{c}=0.473$, with the spatial structure of its flow displayed in figure $2(a, b)$. Note that there are no noticeable differences between the numerical solution and the corresponding analytical solutions (4.23)(4.25) when they are plotted. When $\operatorname{Pr}$ increases, the critical wavenumber $m_{c}$ may decrease at the expense of increasing the radial complexity of the flow. At $\operatorname{Pr}=0.025$, the asymptotic expressions (4.21)-(4.22) give rise to $R_{c}=20.4, m_{c}=4, \sigma_{c}=0.248$ with $\sigma_{0}=0.2634$ and six nodes in the radial direction, whose spatial structure is shown in figure $2(c, d)$. The corresponding numerical solution is characterized by $R_{c}=21.6, m_{c}=4, \sigma_{c}=0.247$, showing nearly the identical spatial structure. When $\mathrm{Pr}$ increases to about 0.70 at $E=10^{-4}$, as shown in figure 1, the asymptotic solution given by (4.21), which is still mathematically valid and gives a satisfactory approximation to the problem, ceases to be physically relevant because the walllocalized convection given by (4.26)-(4.28) becomes preferred. A typical wall-localized solution at $\operatorname{Pr}=1.0$ for $E=10^{-4}$ and $\Gamma=2$ is shown in figure 2(e,f). In this case, the asymptotic analysis gives $R_{c}=63.6, m_{c}=9.56$ and $\sigma_{c}=0.0020$, while the numerical analysis yields $R_{c}=62.6, m_{c}=10$ and $\sigma_{c}=0.0024$. Moreover, the agreement between the asymptotic and numerical analysis is expectedly better for smaller values of $E$. For example, at $E=10^{-5}$ with $\Gamma=2$ and $\operatorname{Pr}=0.01$, the asymptotic expressions (4.21)-(4.22) yield $R_{c}=15.0, m_{c}=2, \sigma_{c}=0.150$ while the numerical analysis gives $R_{c}=15.4, m_{c}=2, \sigma_{c}=0.150$.

Our analysis reveals that there exist three different regimes of convection in a rotating cylinder at a given small $E$. The first is the regime of inertial convection at very small $\operatorname{Pr}$, i.e. $0 \leqslant \operatorname{Pr} / E \leqslant O(1)$. As displayed in the left portion of figure 1 for $0 \leqslant \operatorname{Pr} \leqslant 10^{-4}$, as well as indicated by the asymptotic expression (4.21), the critical Rayleigh number $R_{c}$ is nearly independent of $\operatorname{Pr}$ with $R_{c}=O\left(E^{1 / 2}\right)$. In this regime, the temperature $\Theta$ is marked by approximately the same phase as that of the vertical flow $u_{z}$ in the convective heat transport. But this situation is changed when $\operatorname{Pr} / E>O(1)$, which is shown in the middle portion of figure 1 for $10^{-4}<\operatorname{Pr}<10^{-1}$. Although the inertial effect is still predominant, the temperature $\Theta$ is marked by a large phase shift comparing to the vertical flow $u_{z}$ and, consequently, the $R_{c}$ increases significantly with increasing $\mathrm{Pr}$. In this moderate- $\mathrm{Pr}$ regime, the asymptotic expression (4.21) indicates that there exist no simple asymptotic relations between $R_{c}$ and $E$ or between $R_{c}$ and $\mathrm{Pr}$. It is also in this regime that convection pattern is not only highly complex but also strongly dependent on $\mathrm{Pr}$. This complicated behaviour stems from the existence of a large manifold of two-dimensional inertial modes that may be excited by convective instabilities in this regime, indicating how difficult any numerical attempt to pinpoint its coherent structure will be. For larger values of $\mathrm{Pr}$, which is shown in the right portion of figure 1 for $\operatorname{Pr}>10^{-1}$, the strong viscous effect resulting from the walllocalized boundary-layer flow is dynamically dominant, leading to the relatively simple asymptotic dependence. Our calculations for different values of $E$ suggest that this picture is qualitatively correct for $\Gamma=O(1)$ at any given small Ekman number $E$.

\section{Summary and remarks}

The problem of convection in a rotating circular cylinder heated from below and rotating about a vertical axis has been studied as a paradigm for understanding the general dynamics of rotating convection. We have undertaken both numerical and 
analytical studies of convective instabilities under experimental boundary conditions, showing that the instabilities are either of inertial-wave type or of wall-localized type. In the asymptotic study, the primary assumption is that the convective flow at small or moderate $\mathrm{Pr}$ can be represented by an inertial-wave mode. In the numerical study, we have solved the partial differential equations governing the convection problem. Agreement between the analytical and numerical solutions has been shown to be satisfactory.

There are physical and mathematical consequences of our asymptotic analysis for convection in rotating cylinders. Physically speaking, when a cylinder rotates rapidly $(E \ll 1)$, the fundamental dynamics of fluid motions can be intuitively illustrated by the Proudman-Taylor theorem stating that infinitesimal steady motions in rotating inviscid fluids are two-dimensional with respect to the direction of $\hat{z}$. It follows that $\partial u_{z} / \partial z=0$ and that, upon applying the boundary condition on the top and bottom of a cylinder, we must conclude that $u_{z}=0$ within the cylinder. In other words, convection cannot take place: the effect of rotation strongly constrains and stabilizes the system. The constraint must be broken so that convection can occur. Our asymptotic analysis shows that it can be broken either by inertial effects for fluids with small Prandtl numbers or by viscous effects for fluids with large Prandtl numbers in connection with the boundary-layer-type convection. Mathematically speaking, the basic assumption that the convection velocity at leading order for small or moderate $\mathrm{Pr}$ can be represented by an inertial-wave mode implies that the present stability analysis is quite different from the usual one such as that for the Rayleigh-Bénard problem. In our stability analysis, it is not a question of simply finding a wavenumber $m$ that minimizes the Rayleigh number and then determines the most unstable convection mode. It is a question of finding the two-dimensional structure of a leading-order flow that gives rise to the minimum Rayleigh number. This explains why it is so difficult to elucidate the parametric dependence of the problem numerically. Moreover, since the asymptotic formulas can be readily evaluated, they would provide a helpful guidance for the experimental studies of convection in rotating circular cylinders.

This study represents the first asymptotic analysis for convective instabilities in a rotating cylinder with experimental boundary conditions for small and moderate Prandtl numbers. It is significant to note that the two sets of asymptotic formulas given in $\S 4$ cover the whole parameter regime of the problem: all values of the aspect ratio $\Gamma$ and the Prandtl number $P r$ at any fixed small Ekman number E. Especially, it is somewhat surprising that the seemingly incomprehensible and highly complicated convection revealed numerically, for example, by Goldstein et al. $(1993,1994)$ can be satisfactorily described by several relatively simple analytical formulas.

Keke Zhang is supported by UK PPARC/NERC grants and Xinhao Liao is supported by NSFC/10633030, STCSM/08XD14052 and CAS grants. The numerical computation is supported by SSC. We would like to thank Professor F. H. Busse for his helpful discussions.

\section{REFERENCES}

Aurnou, J. M., Andreadis, S., Zhu, L. \& Olson P. L. 2003. Experiments on convection in Earth's core tangent cylinder. Earth Planet. Sci. Lett. 212, 119-134.

Busse, F. H. \& Simitev R. 2004. Inertial convection in rotating fluid spheres. J. Fluid Mech. 498, 23-30.

Daniels, P. G. 1980. The effect of centrifugal acceleration on axisymmetric convection in a shallow rotating cylinder or annulus. J. Fluid Mech. 99, 65-84. 
Goldstein, H. F., Knobloch, E., Mercader, I. \& Net, M. 1993. Convection in a rotating cylinder. Part 1. Linear theory for moderate Prandtl numbers. J. Fluid Mech. 248, 583-604.

Goldstein, H. F., Knobloch, E., Mercader, I. \& Net, M. 1994. Convection in a rotating cylinder. Part 2. Linear theory for small Prandtl numbers. J. Fluid Mech. 248, 583-604.

Herrmann, J. \& Busse, F. H. 1993. Asymptotic theory of wall-localized convection in a rotating fluid layer. J. Fluid Mech. 255, 183-194.

Kuo, E. Y. \& Cross, M. C. 1993. Travelling-wave wall states in rotating Rayleigh-Benard convection. Phys. Rev. E 47, 2245-2248.

LiaO, X., Zhang, K. \& Chang, Y. 2006. On boundary-layer convection in a rotating fluid layer. J. Fluid Mech. 549, 375-384.

MARQuÉS, F. 1990. On boundary conditions for velocity potentials in confined flows: application to Couette flow. Phys. Fluids A 2, 729-737.

Net, M., Carcia, F. Sanchez, J. On the onset of low-Prandtl-number convection in rotating spherical shells: non-slip boundary conditions. J. Fluid Mech. 601, 317-337.

Plaut, E. 2003. Nonlinear dynamics of travelling Rayleigh-Bénard convection: effects of the boundary conditions and of the topology. Phys. Rev. E 67, 046303, 1-11.

Young, R. M. B. \& READ, P. L. 2008. Breeding and predictability in the baroclinic rotating annulus using a perfect model. Nonlinear Process. Geophys. 15, 469-487.

ZhANG, K. 1995. On coupling between the Poincare equation and the heat equation: non-slip boundary condition. J. Fluid Mech. 284, 239-256.

Zhang, K. \& LiaO, X. 2008. On the initial value problem in a rotating circular cylinder. J. Fluid Mech. 610, 425-443.

Zhang, K., LiaO, X. \& Busse, F. H. 2007. Asymptotic theory of inertial convection in a rotating cylinder. J. Fluid Mech. 575, 449-471.

Zhong, F., Ecke, R. E. \& Steinberg, V. 1991. Asymmetric modes and the transition to vortex structure in rotating Rayleigh-Benard convection. Phys. Rev. Lett. 67, 2473-2476. 